\title{
A National Multicenter Study on overall survival in elderly metastatic castrate-resistant prostate cancer patients treated with Radium-223
}

\author{
Viviana Frantellizzi ${ }^{1}$ (1) $\cdot$ Fabio Monari ${ }^{2} \cdot$ Manlio Mascia $^{3} \cdot$ Renato Costa $^{4} \cdot$ Giuseppe Rubini $^{5} \cdot$ Angela Spanu $^{6}$. \\ Alessio Farcomeni ${ }^{7}$. Elisa Lodi Rizzini ${ }^{8} \cdot$ Luca Cindolo $^{9} \cdot$ Alessandra Murabito $^{4}$ - Valentina Lavelli ${ }^{5}$. Susanna Nuvoli ${ }^{6}$. \\ Laura Cosma $^{10} \cdot$ Valeria Dionisi $^{2} \cdot$ Anna Giulia Nappi $^{5} \cdot$ Marco Andreola $^{10} \cdot$ Giuseppe De Vincentis $^{10}$
}

Received: 21 February 2020 / Accepted: 17 April 2020 / Published online: 1 May 2020

(c) Springer Nature Switzerland AG 2020

\begin{abstract}
Background Radium-223 prolongs overall survival (OS) and delays time to the first symptomatic skeletal events in patients with symptomatic metastatic castration-resistant prostate cancer (mCRPC). There is a lack of evidence on the safety and efficacy of Radium-223 treatment in the very elderly population.

Aims Aim of this multicentre study is to analyze mCRPC patients treated with Radium-223 in terms of OS and to assess whether there are differences between young and elderly, as well as to verify efficacy and safety in patients $\geq 75$ years of age. Methods 430 mCRPC patients of six Italian Centres were analyzed in this multicenter retrospective study. At baseline and after each cycle were collected clinical and diagnostic patients' parameters. The whole cohort was divided into two groups based on the age of the patients ( $<75$ years old and $\geq 75$ years old).

Results $47 \%$ of the patients were $<75$ years old and $53 \%$ were $\geq 75$ years old. The primary outcome, OS, does not show significant differences between the two subgroups if other basal parameters are considered. Considering clinical covariates in univariate models $(p<0.05)$ several clinical aspects have an impact on OS, except for age $(p=0.072)$. Age continues to have no significant impact on the OS $(p=0.274)$ even in multivariate models in the two groups. The toxic effects are similar in the two groups.

Conclusions Radium-223 prolongs survival in both younger and older patients at the same baseline condition and is a good option in the symptomatic mCRPC setting compared to other agents.
\end{abstract}

Keywords mCRPC $\cdot$ Elderly $\cdot$ Overall survival $\cdot$ Radium-223 $\cdot$ Prostate cancer

Viviana Frantellizzi

viviana.frantellizzi@uniroma1.it

1 Department of Molecular Medicine Sapienza, "Sapienza" University of Rome, Viale Regina Elena 324, 00161 Rome, Italy

2 Radiation Oncology Center, S.Orsola-Malpighi Hospital, Bologna, Italy

3 Unit of Nuclear Medicine, "Spirito Santo" Hospital, Pescara, Italy

4 Unit of Nuclear Medicine, Biomedical Department of Internal and Specialist Medicine, University of Palermo, Palermo, Italy

5 Nuclear Medicine Department, University of Bari "Aldo Moro", Bari, Italy
6 Unit of Nuclear Medicine, Department of Medical Surgical and Experimental Sciences, University of Sassari, Sassari, Italy

7 Department of Economics \& Finance, University of Rome "Tor Vergata", Rome, Italy

8 Nuclear Medicine Unit, S.Orsola-Malpighi Hospital, Bologna, Italy

9 Department of Urology, "Villa Stuart" Private Hospital, Rome, Italy

10 Department of Radiological Sciences, Oncology and Anatomical Pathology Sapienza, "Sapienza" University of Rome, Rome, Italy 


\section{Introduction}

Prostate cancer $(\mathrm{PCa})$ represents one of the most important types of cancer by incidence [1]. The lethal stage of the disease is reached when cancer does not respond to hormone therapy anymore and frequently metastasizes to the bone [2-4]. Bone metastasis deteriorates the patient's clinical condition and quality of life (QoL) as well [5]. Symptoms reported are bone fractures, pain, spinal cord compression, hypercalcemia, impaired mobility with a significant impact on mortality and morbidity [6]. A considerable percentage of patients present this disease in the elderly age and older men are mostly affected by PCa. Treatments used for pain relief and involved in the delay of skeletal events are bisphosphonates, denosumab, external beam radiation therapy and radioisotope treatments [7-11]. In the last few years, Radium-223 treatment took on relevance in patients with metastatic castration-resistant prostate cancer (mCRPC) [12]. This condition, defined as a serum testosterone level of $50 \mathrm{ng} / \mathrm{ml}$ or lower, in biochemical and/or radiological progression after androgen deprivation therapy (ADT), is developed by $10 \%$ of $\mathrm{PCa}$ patients and more than $90 \%$ of them have skeletal metastases. Radium-223 is an alpha particle emitter with a halflife of 11.4 days that produce high-energy particles, inducing double-stranded breaks in DNA, with a tissue range of fewer than $100 \mu \mathrm{m}$ [13]. As a calcium mimetic, it targets regions with high osteoblastic activity such as bone metastases, inducing local cytotoxic effect with limited toxicity to surrounding tissue and marrow and minimal hematological adverse effects. Approved in 2013 by the Federal and drug administration (FDA) for treatment of mCRPC patients with symptomatic bone metastases, Radium-223 improves overall survival (OS) with low myelosuppression rates and few adverse events as shown in the randomized phase 3 Radium-223 ALSYMPCA trial [14]. In this trial, comparing patients receiving Radium-223 with those who received placebo, OS was significantly longer (median survival: 14.9 vs 11.3 months; hazard ratio [HR] 0.70 ; 95\% CI $0.58-0.83 ; p<0.0001$ ) and significantly longer time to first symptomatic skeletal event (SRE) (15.6 vs 9.8 months) generally correlated with significant morbidity and mortality, impairing QoL [15] and leading to neurological damage, in the case of spinal cord compression. Toxicities reported were generally mild and comparable to placebo: in $13 \%$ of Radium-223-treated patients grade 3-4 anemia, in 6\% grade 3-4 thrombocytopenia, grade 3-4 neutropenia in 3\% of treated patients (far more favorable than that of docetaxel or cabazitaxel; $3 \%$ for Radium-223 vs $82 \%$ for cabazitaxel) [16] and occasional cases of fatigue, nausea and loose stools. Furthermore, Radium-223 has to be considered next to Abiraterone or
Enzalutamide and it is able to provide pain relief, determining an improvement in QoL $[17,18]$. Lack of evidence regarding the efficacy of Radium-223 treatment in the elderly population and lack of literature data about very elderly patients, often excluded from clinical trials, has led to evaluate this particular aspect in this study [19]. Nowadays, elderly age must be reconsidered because of the elongation of life expectancy due to improvement and discoveries in scientific and medical fields. In light of the above, this assessment has led SIGG (Italian Society of Gerontology and Geriatrics), in the $2018^{\text {th }}$ National Italian Congress, to shift the threshold of elderly age from 65 to 75 years old, also based on many studies on this topic [20]. Moreover, some aspects should be taken into account in as much as elderly men with mCRPC should be managed not according to the mere chronological age, that cannot include and describe the wide heterogeneity present in each individual aging process, but according to the global health status [21]. The purpose of this multicentre study is to analyze mCRPC patients treated with Radium-223 and to evaluate if there are differences between young and old, as well as to verify efficacy and safety in the category of patients $\geq 75$ years old.

\section{Materials and methods}

This retrospective multicentre study included consecutive mCRPC patients treated with Radium-223 between July 2015 and July 2019 in six Italian centers. Inclusion criteria were at least 2 symptomatic skeletal metastases detected by ${ }^{99 m}$ Tc-HDP bone scintigraphy, no known visceral metastases at contrast-enhanced CT scan, except for malignant lymphadenopathy with less than $3 \mathrm{~cm}$ in the short-axis diameter and an Eastern Cooperative Oncology Group (ECOG) performance status (PS) score of 0-2 [22]. Exclusion criteria were impaired kidney and liver function and the presence of inflammatory bowel disease (IBD). This study was approved by the local Ethical Committee of all participating centers and was performed in accordance with the ethical standards of the 1964 Declaration of Helsinki and its later amendments. All patients signed a written Informed Consent. Radium-223 was administered, according to the Italian label authorization at that time in force, intravenously, in 6 cycles every 28 days at the activity of $55 \mathrm{kBq} / \mathrm{kg}$ [23]. At baseline and before each cycle were evaluated and collected the following patient's parameters: blood values [hemoglobin $(\mathrm{Hb})$, platelets (PLT), absolute neutrophil counts (ANC)], prostatespecific antigen (PSA), total alkaline phosphatase (tALP), lactate dehydrogenase (LDH) and clinical outcome (in particular pain by Numeric Rating Scale(BPI) and ECOG-PS). Therefore, patients were submitted to bone scintigraphy just before starting treatment, after the third cycle and at the 
end of the treatment to assess baseline skeletal metastasis and, eventually, improvement or worsening of health condition. QoL was evaluated with an EORTC questionnaire [24] filled out by the patients and allowed us to calculate and report the Narcotic Score relying on the medical type of pharmaceutical taken to relieve it (analgesic, mild narcotic, strong narcotic) and on the frequency. The whole cohort was divided into two groups based on the age of the patients ( $<75$ years old and $\geq 75$ years old). The main endpoint was the OS, which was considered in the period between the first administration of Radium-223 and the statistical analysis.

\section{Statistical analysis}

Data are expressed as mean \pm standard deviation or median \pm IqR as appropriate. Categorical data are expressed as counts or percentages. Means were compared by Student's two-sample $T$ test with Welch correction or Mann-Whitney test, accordingly. Association among categorical variables was evaluated by means of Chi squared test or Fisher test for binary variables. Survival distributions were estimated using the Kaplan-Meyer product-limit estimator and compared using the log-rank test. The association of predictors with OS was evaluated by means of univariate Cox regression models. A multivariable Cox regression model was then selected by forcing the age variable (which is a target of our analysis) and using a stepwise forward procedure based on Akaike Information Criterion to include other predictors. Variance Inflation Factors were checked for collinearity and residual analysis was used to assess the proportionality of hazards assumption. The significance level was set at 5\%. All analyses were conducted using the R software, version 3.5.1.

\section{Results}

430 mCRPC patients treated with Radium 223 were analyzed. Patients baseline characteristics are reported in Table 1. In this cohort, the mean age was $74.1 \pm 1.9$ years. The following parameters were collected at baseline and are reported in Table 1: blood values (Hb, PLT, ANC), PSA, tALP and LDH. The mean follow-up period from the first cycle of Radium-223 treatment to the date of the analysis or death was $12.7 \pm 8.8$ months. 157 patients (36.5\%) died during the study period and 273 (63.5\%) were still alive at the time of analysis. The reasons for Radium-223 treatment drop-out before the end of all 6 cycles were progression disease, bone marrow failure, SRE and death. $47 \%$ of the patients were $<75$ years old (YOUNGER GROUP) and 53\% $\geq 75$ years old (ELDERLY GROUP), defining elderly patients equal or older than 75 years. The baseline characteristics of the patients divided into the two subgroups based on
Table 1 Baseline patients' characteristics

\begin{tabular}{|c|c|c|}
\hline Baseline variable & Patients $(n=430)$ & $\%$ \\
\hline \multicolumn{3}{|l|}{ Age (years) } \\
\hline Mean (SD) & $74.1(1.9)$ & \\
\hline \multicolumn{3}{|l|}{ Gleason score } \\
\hline Mean (range) & $7.9(5-10)$ & \\
\hline 5 & 3 & 0.7 \\
\hline 6 & 22 & 5.1 \\
\hline 7 & 108 & 25.1 \\
\hline 8 & 99 & 23 \\
\hline 9 & 110 & 25.6 \\
\hline 10 & 9 & 2.1 \\
\hline Unknown & 79 & 18.4 \\
\hline \multicolumn{3}{|l|}{ Skeletal burden } \\
\hline $0-6$ mets & 66 & 15.3 \\
\hline $6-20$ mets & 266 & 61.9 \\
\hline$\geq 20$ mets & 98 & 22.8 \\
\hline \multicolumn{3}{|c|}{ Number of previous systemic treatments } \\
\hline Mean (SD) & $1.58(1.2)$ & \\
\hline 0 & 65 & 15.1 \\
\hline 1 & 117 & 27.2 \\
\hline 2 & 74 & 17.2 \\
\hline$\geq 3$ & 78 & 18.1 \\
\hline Unknown & 96 & 22.3 \\
\hline Baseline PSA, mean (SD) & $198(655) \mathrm{ng} / \mathrm{ml}$ & \\
\hline Baseline tALP, mean (SD) & 267 (308) U/1 & \\
\hline Baseline $\mathrm{Hb}$, mean (SD) & $12.2(2.5) \mathrm{g} / \mathrm{dl}$ & \\
\hline Baseline LDH, mean (SD) & $359(221) \mathrm{U} / \mathrm{l}$ & \\
\hline Baseline PLT, mean (SD) & $240.4(94.5) 10^{3} / \mathrm{mmc}$ & \\
\hline Baseline ANC, mean (SD) & $4.6(1.8) 10^{3} / \mu 1$ & \\
\hline
\end{tabular}

age ( $<75$ years old and $\geq 75$ years old) are compared in Table 2. YOUNGER GROUP median age was 68 years (IqR 8, range 50-74), while ELDERLY GROUP median age was 80 years (IqR 6, range 75-92). An overview of statistical analysis has been reported in Table 3. The primary clinical outcome, OS, does not show significant differences between the two subgroups if other basal parameters are considered. Indeed, considering clinical covariates in univariate models $(p<0.05)$ several clinical aspects have an impact on OS, except for age $(p=0.072)$ (See Kaplan-Meier curves in Fig. 1). Age continues to have no significant impact on the OS $(p=0.274)$ even in multivariate models in the two groups. This means that for the same basal tALP, Hb and LDH there is no effect of any parameter on survival, in other words, the data show no evidence that there is a difference between above and below 75 years age groups. In the two groups, there are not many differences both in terms of the number of systemic therapies received before Radium-223 and in terms of the number of cycles of Radium-223 received 
Table 2 Age groups baseline characteristics

\begin{tabular}{|c|c|c|c|}
\hline & $\begin{array}{l}\text { YOUNGER GROUP } \\
(<75 \text { years })(n=200)\end{array}$ & $\begin{array}{l}\text { ELDERLY GROUP } \\
(\geq 75 \text { years })(n=230)\end{array}$ & $p$ value \\
\hline \multicolumn{4}{|l|}{ Gleason score } \\
\hline Mean (SD) & $8.02(0.98)$ & $7.79(1.05)$ & 0.032 \\
\hline \multicolumn{4}{|l|}{ ECOG PS } \\
\hline 0 & $91(46 \%)$ & $90(39 \%)$ & \multirow[t]{4}{*}{0.46} \\
\hline 1 & $68(34 \%)$ & $83(36 \%)$ & \\
\hline 2 & $38(19 \%)$ & $51(22 \%)$ & \\
\hline 3 & $2(1 \%)$ & $5(2 \%)$ & \\
\hline \multicolumn{4}{|l|}{ Brief Pain Inventory (BPI) pain score } \\
\hline Low $(0-3)$ & $62(31 \%)$ & $78(34 \%)$ & \multirow[t]{3}{*}{0.78} \\
\hline Intermediate (4-7) & $102(51 \%)$ & $110(47.8 \%)$ & \\
\hline Severe $(8-10)$ & $36(18 \%)$ & $42(18.2 \%)$ & \\
\hline \multicolumn{4}{|l|}{ Progression disease } \\
\hline Radiological & $3(1.5 \%)$ & $5(2.1 \%)$ & \multirow[t]{3}{*}{0.92} \\
\hline Biochemical & $12(6 \%)$ & $15(6.5 \%)$ & \\
\hline Clinical (pain) & $1(0.5 \%)$ & $1(0.43 \%)$ & \\
\hline $\mathrm{N}$ of Radium-223 treatment drop-out & $75(37.5 \%)$ & $83(36.3 \%)$ & 0.7 \\
\hline \multicolumn{4}{|l|}{$\mathrm{N}$ of Radium-223 administered cycles } \\
\hline Median & 6 & 6 & \\
\hline 1 & $12(6 \%)$ & $15(6.5 \%)$ & \multirow{6}{*}{0.7} \\
\hline 2 & $10(5 \%)$ & $17(7.5 \%)$ & \\
\hline 3 & $18(9 \%)$ & $13(5.6 \%)$ & \\
\hline 4 & $15(7.5 \%)$ & $21(9.1 \%)$ & \\
\hline 5 & $24(12 \%)$ & $20(8.7 \%)$ & \\
\hline 6 & $121(60.5 \%)$ & $144(62.6 \%)$ & \\
\hline \multicolumn{4}{|l|}{$\mathrm{N}$ of previous systemic treatments } \\
\hline Median (IQR) & $1(1)$ & $1(1)$ & 0.51 \\
\hline \multicolumn{4}{|l|}{ Types of previous systemic treatments } \\
\hline Taxanes & $138(69 \%)$ & $120(52 \%)$ & \multirow[t]{2}{*}{0.11} \\
\hline New hormonal agents & $135(67.5 \%)$ & $157(68 \%)$ & \\
\hline $\begin{array}{l}\text { Worst hematological toxicities (blood } \\
\text { transfusion or anemia G4) }\end{array}$ & 7 & 8 & 1 \\
\hline Skeletal-related events (SREs) & 5 & 3 & 0.58 \\
\hline \multicolumn{4}{|l|}{ Skeletal burden } \\
\hline $0-6$ mets & $53(26 \%)$ & $45(20 \%)$ & \multirow[t]{3}{*}{0.22} \\
\hline $6-20$ mets & $119(60 \%)$ & $147(64 \%)$ & \\
\hline$\geq 20$ mets & $28(14 \%)$ & $38(16 \%)$ & \\
\hline Baseline PSA ng/ml, median (IQR) & $49.22(181.20)$ & $51.30(126.94)$ & 0.37 \\
\hline Baseline tALP U/l, median (IQR) & $148(196.5)$ & $141(194.5)$ & 0.66 \\
\hline Baseline LDH U/1, median (IQR) & $308.5(217.5)$ & $305.0(202.5)$ & 0.65 \\
\hline Baseline $\mathrm{Hb}$ g/dl, mean (SD) & $12.24(1.53)$ & $12.15(1.49)$ & 0.52 \\
\hline Baseline PLT $10^{3} / \mathrm{mmc}$, median (IQR) & $221(86.0)$ & $220(85.5)$ & 0.99 \\
\hline Baseline ANC $10^{3} / \mu 1$, mean (SD) & $4.66(1.94)$ & $4.60(1.65)$ & 0.76 \\
\hline
\end{tabular}

by the patients analyzed. Moreover, if we consider the toxic effects, understood as hematological or SRE, as we note in Table 3 , they are few and, in any case, similar in the two groups, continuing to guarantee the safety of Radium-223 even in very elderly patients.

\section{Discussion}

Approximately $10 \%$ of PCa patients develop a CRPC and more than $90 \%$ of them have skeletal metastases which 
Table 3 Univariate and multivariable analysis of OS in relation to baseline variables

\begin{tabular}{lllll}
\hline Clinical covariates & Univariate models HR (95\% CI) & $p$ value & $\begin{array}{l}\text { Multivariable model } \\
\text { HR (95\% CI) }\end{array}$ & $p$ value \\
\hline Age $\geq 75$ & $1.246(0.981-1.583)$ & 0.072 & $1.433(0.752-2.734)$ & 0.274 \\
BMI & $0.948(0.918-0.979)$ & 0.001 & & \\
Gleason score & $0.928(0.818-1.053)$ & 0.246 & & \\
ECOG PS & $1.534(1.325-1.775)$ & 0.000 & & 0.000 \\
Baseline Hb & $0.722(0.663-0.787)$ & 0.000 & $0.675(0.542-0.841)$ & \\
Baseline PSA & $0.001(1.001-1.001)$ & 0.000 & & 0.001 \\
Baseline tALP & $1.001(1.001-1.002)$ & 0.000 & $1.002(1.001-1.003)$ & 0.000 \\
Baseline LDH & $0.003(1.002-1.004)$ & 0.000 & $1.003(1.002-1.004)$ & \\
Baseline PLT & $1.001(1.000-1.003)$ & 0.148 & & \\
Baseline ANC & $1.098(1.024-1.178)$ & 0.009 & & \\
\hline
\end{tabular}

* Age $\geq 75$ is a categorical dummy variable, everything else was used as a continuous predictor

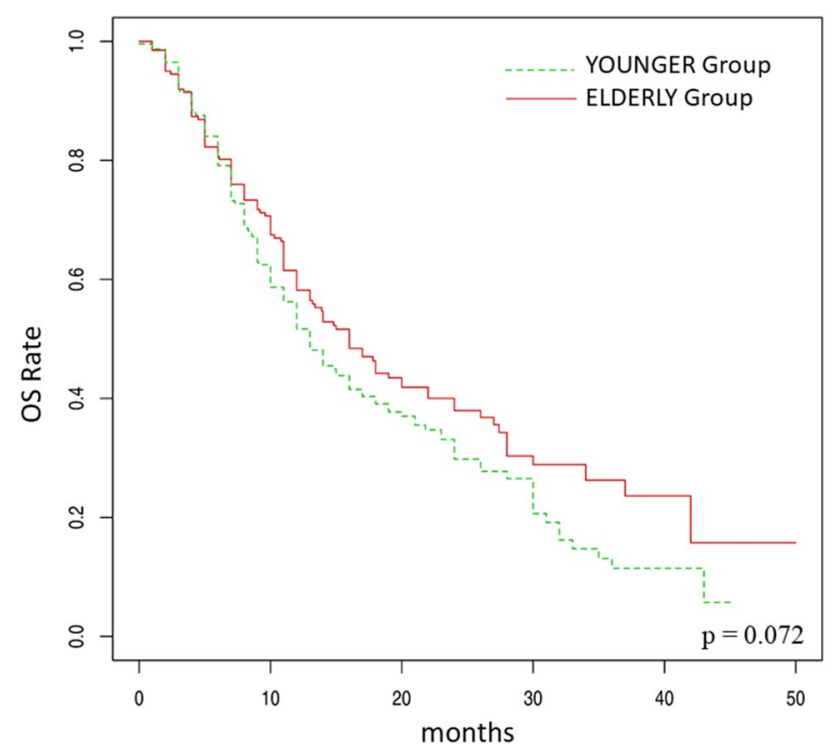

Fig. 1 Kaplan-Meier analysis shown the YOUNGER and the ELDERLY groups. The curve showed no difference in overall survival between the two groups

are the cause of reduced Qol and responsible for disability $[17,25]$. Several new drugs have been introduced and approved for the management of mCRPC patients such as immunotherapeutic agent (sipuleucel-T), hormonal treatments (abiraterone acetate and enzalutamide), taxane agent (docetaxel and cabazitaxel) and a radiopharmaceutical (Radium-223). All these pharmaceuticals are able to improve OS [26]. A recent challenge is represented by the search for the most suitable drug sequence for elderly and frail patients. In fact, this particular category shows vulnerability to adverse effects and often develop resistance mechanisms to taxane chemotherapy as well as hormonal therapy [27, 28]. Moreover, factors other than age should be considered when treating mCRPC, for example, the reduction of functional reserves that physiologically decline with age. This leads to an increased risk of interactions between drugs taken for comorbidities. As the International Society of Geriatric Oncology (SIOG) PCa task force has recently updated, the appropriate management of elderly patients should be conducted not according to chronological age but to the individual health status [21]. Validated tools that assess vulnerability in elderly patients with cancer, such as the G8, the CISR-G scale, the daily life activity ADL and a comprehensive geriatric assessment (CGA) would be useful for initial health assessment decisions for fragile or disabled patients or with severe comorbidities. In light of the above, the recent SIOG guidelines classify oncologic PCa patients according to health status into four groups (fit, frail, disabled, terminally ill) accompanied by different cancer treatment plan. International recommendations underline that fit elderly patients should be treated as younger patients. Both fit and frail symptomatic patients with mCRPC should receive first-line docetaxel. For fit patients at progression after docetaxel, cabazitaxel and hormonal agents are appropriate whereas for those who are disabled or with severe comorbidities or reluctant to receive chemotherapy, they should receive hormonal agents [21]. Because of potential cross-resistance between androgen receptor-targeted agents, if treatment with a novel hormonal agent fails, there is a consensus that drugs with a different mechanism of action (taxanes or Radium-223) should be taken in consideration [2]. In particular, SIOG has recently addressed the use of radiopharmaceuticals and such a perspective is important because compared with cytotoxic and cytostatic drugs, therapeutic radiopharmaceuticals are less toxic alternatives [21]. Also, in this study, we have shown that the toxic effects due to Radium-223 are few and however similar both in the very elderly and in the younger patients. The number of SRE is also comparable. Several studies demonstrated a correlation between the number of Radium-223 administration delivered and OS. Patients 
who completed the planned six cycle had a reduced risk of death, reduced progression, SRE and reduced development of bone marrow failure (BMF) compared to patients who didn't receive all six doses [29]. Furthermore, patients undergoing Radium-223 treatment and previous radical prostatectomy (RP) and/or ablative external beam radiotherapy (EBRT) have shown an improvement in OS rather than those who did not [30]. Moreover, several studies showed that patients Radium-223 treated and who had never undergone previous chemotherapy, had a much lower hematological toxicity than those who had previously been treated with chemotherapy. In this contest, to guarantee a better toxicity profile, postponing chemotherapy and anticipating the treatment with Radium-223 it may be appropriate, although European Medicines Agency (EMA) recommended that Radium-223 can only be administered in progression disease patients after at least two previous systemic therapy lines for mCRPC. Several prognostic factors associated with survival after Radium-223 have been presented in most previous retrospective studies like ECOG, tALP, Hb value, previous systemic treatments but anyone identified as a predictive clinical marker for OS [31-34]. However there is a need for a multidimensional clinical and laboratory evaluation to select patients suitable to receive benefit from Radium-223 treatment: the 3-PS baseline evaluation proposed by another study, strongly associated with OS of the patients mCRPC Radium-223 treated, is a promising tool useful for identifying them [35]. In this study, we assessed the impact of age on OS in MCRPC patients treated with Radium-223 and, comparing younger and older patients, data analysis showed that the two groups have similar OS at similar baseline conditions of the patient. Therefore, our recent clinical experience shows that age alone is not a determining factor of survival outcomes among this category of patients. There is no evidence that OS should vary with age, conditional on basal characteristics. Rephrasing, our evidence is that two patients with equal basal tAPL, Hb and LDH values can be expected to have the same survival regardless of the presenting age. The results of this study are in line with a post hoc analysis of ALSYMPCA that examined men by age and in which treatment with Radium-223 resulted in a significant prolonged OS and with similar or fewer adverse events of the men in the group aged $\geq 75$ than those in the younger age group [36]. It should be emphasized that in the population of this study, the number of cycles of Radium-223 received is similar in the two subgroups analyzed, as well as the number of systemic therapies received before Radium-223, therefore even this, which could have been a limit in the analysis, was overcome by the almost homogeneity of the groups analyzed. The most important prognostic parameter seems to be the general baseline health condition and not the chronological age. Taking in consideration that age does not affect the tolerability or efficacy of Radium-223 treatment, this radiopharmaceutical could be considered a safer option before other therapies characterized by high rates of toxicities and a curable approach should be considered for the patient according to their general condition, independently of their age [37]. To maximize survival and QoL of these patients and to minimize treatment-related morbidity, there is the need of a CGA and critical considerations as a functional reserve, comorbidities and life expectancy should be done to choose the best therapeutic modalities and to determine the optimal sequencing or combination of the available treatments [38].

Since this study was done considering only the inclusion criteria for the use of Radium-223, there are several limitations. The retrospective nature of the study does not allow for many analyzes that can be evaluated in the future with a prospective analysis. In addition to the aforementioned analysis of the comorbidities of mCRPC patients, it would be interesting in the near future to study the impact of therapeutic sequences of each patient received before the administration of Radium-223, which in this study we could not analyze because of the too small sample. Since this oncological work was born for the evaluation of the OS, the part of the geriatric evaluation was not carried out, but it would also be interesting in a prospective study to administer Q8 or CGA to all patients and overcome the limit of this study.

All this should, therefore, be enclosed in a multidimensional evaluation of the patient, which guarantees good conditions before the administration of Radium-223 to increase its benefits, without considering the chronological age.

\section{Conclusions}

The management of individual elderly patients should not be based on age but on their health status. As observed in this study, Radium-223 may prolong survival in both younger and older patients at the same baseline condition and is a good option in the mCRPC setting compared to other agents, especially for men unsuitable for cytotoxic chemotherapy. In light of the above, Radium-223, better tolerated and characterized by low rates of adverse effects, is suitable for the treatment of younger and even more for older, frails and vulnerable patients.

Funding No funding has been received for this paper

\section{Compliance with ethical standards}

Conflicts of interest The Authors declare that they have no conflict of interest. 
Ethics approval This study was approved by the local Ethical Committee and was performed in accordance with the ethical standards of the 1964 Declaration of Helsinki and its later amendments.

Research involving Human Participants and/or Animals This article does not contain any studies with animals performed by any of the authors.

Informed consent Informed consent was obtained from all individual participants included in the study.

Consent for publication Not applicable.

\section{References}

1. Siegel RL, Miller KD, Jemal A (2018) Cancer statistics. CA Cancer J Clin 68:7-30. https://doi.org/10.3322/caac.21442

2. Sciarra A, Gentilucci A, Silvestri I et al (2019) Androgen receptor variant 7 (AR-V7) in sequencing therapeutic agents for castratrion resistant prostate cancer: a critical review. Medicine 98:e15608. https://doi.org/10.1097/md.0000000000015608

3. Ricci M, Frantellizzi V, Bulzonetti N et al (2018) Reversibility of castration resistance status after Radium-223 dichloride treatment: clinical evidence and review of the literature. Int J Radiat Biol. https://doi.org/10.1080/09553002.2019.1558301

4. Mohler JL, Antonarakis ES, Armstrong AJ et al (2019) Prostate Cancer, Version 2. 2019, NCCN Clinical Practice Guidelines in Oncology. J Natl Compr Cancer Netw 17:479-505. https://doi. org/10.6004/jnccn.2019.0023

5. Sciarra A, Gentilucci A, Salciccia S et al (2018) Psychological and functional effect of different primary treatments for prostate cancer: a comparative prospective analysis. Urol Oncol 36:340. https://doi.org/10.1016/j.urolonc.2018.03.022

6. Jiang W, Ulmert D, Simons BW et al (2018) The impact of age on radium-223 distribution and an evaluation of molecular imaging surrogates. Nucl Med Biol 62-63:1-8. https://doi.org/10.1016/j. nucmedbio.2018.05.003

7. Costa RP, Bordonaro S, Cappuccio F et al (2019) Hematologic toxicity of radium-223 in elderly patients with metastatic Castration Resistant Prostate Cancer: a real-life experience. Prostate Int 7:25-29. https://doi.org/10.1016/j.prnil.2018.08.001

8. Parker C, Heidenreich A, Nilsson S et al (2018) Current approaches to incorporation of radium-223 in clinical practice. Prostate Cancer Prostatic Dis 21:37-47. https://doi.org/10.1038/ s41391-017-0020-y

9. Lutz S, Balboni T, Jones J et al (2017) Palliative radiation therapy for bone metastases: update of an ASTRO Evidence-Based Guideline. Pract Radiat Oncol 7:4-12. https://doi.org/10.1016/j. pro.2016.08.001

10. Shulman RM, Meyer JE, Li T et al (2019) External beam radiation therapy (EBRT) for asymptomatic bone metastases in patients with solid tumors reduces the risk of skeletal-related events (SREs). Ann Palliat Med 8:159-167. https://doi.org/10.21037/ apm.2018.10.04

11. Berg RS, Yilmaz MK, Hoyer M et al (2009) Half body irradiation of patients with multiple bone metastases: a phase II trial. Acta Oncol 48:556-561. https://doi.org/10.1080/02841860802488128

12. Lowrance WT, Murad MH, Oh WK et al (2018) Castration-Resistant Prostate Cancer: AUA Guideline Amendment 2018. J Urol 200:1264-1272. https://doi.org/10.1016/j.juro.2018.07.090

13. Sartor O, Coleman R, Nilsson S et al (2014) Effect of radium-223 dichloride on symptomatic skeletal events in patients with castration-resistant prostate cancer and bone metastases: results from a phase 3, double-blind, randomised trial. Lancet Oncol 15:738746. https://doi.org/10.1016/s1470-2045(14)70183-4

14. Parker C, Nilsson S, Heinrich D et al (2013) Alpha emitter Radium-223 and survival in metastatic prostate cancer. N Engl J Med 369:213-223. https://doi.org/10.1056/nejmoa1213755

15. Roviello G, Gallicchio R, Bozza G et al (2019) Pain predicts overall survival in men with metastatic castration-resistant prostate cancer treated with radium-223. Onco Targets Ther 12:9-13. https ://doi.org/10.2147/ott.S174206

16. Wilson JM, Parker C (2016) The safety and efficacy of radium-223 dichloride for the treatment of advanced prostate cancer. Expert Rev Anticancer Ther 16:911-918. https://doi.org/10.1080/14737 140.2016.1222273

17. Prior JO, Gillessen S, Wirth M et al (2017) Radiopharmaceuticals in the elderly cancer patient: practical considerations, with a focus on prostate cancer therapy: a position paper from the International Society of Geriatric Oncology Task Force. Eur J Cancer 77:127-139. https://doi.org/10.1016/j.ejca.2017.01.030

18. De Vincentis G, Follacchio GA, Frantellizzi V et al (2017) 223Radichloride therapy in an elderly bone metastatic castration-resistant prostate cancer patient: a case report presentation and comparison with existing literature. Aging Clin Exp Res 30:677-680. https://doi.org/10.1007/s40520-017-0826-4

19. Castellucci P, Nanni C, Ambrosini V (2018) Nuclear Medicine Imaging of Prostate Cancer in the Elderly. Semin Nucl Med 48:541-547. https://doi.org/10.1053/j.semnuclmed.2018.07.004

20. Zuo W, Jiang S, Guo Z, Feldman MW et al (2018) Advancing front of old-age human survival. Proc Natl Acad Sci USA 115:11209-11214. https://doi.org/10.1073/pnas.1812337115

21. Droz JP, Albrand G, Gillessen S et al (2017) Management of prostate cancer in elderly patients: recommendations of a task force of the international society of geriatric oncology. Eur Urol 72:521-531. https://doi.org/10.1016/j.eururo.2016.12.025

22. Baldari S, Boni G, Bortolus R et al (2017) Management of metastatic castration-resistant prostate cancer: a focus on radium-223: Opinions and suggestions from an expert multidisciplinary panel. Crit Rev Oncol Hematol 113:43-51. https://doi.org/10.1016/j.critr evonc.2017.03.001

23. Prelaj A, Rebuzzi SE, Buzzacchino F et al (2019) Radium-223 in patients with metastatic castration-resistant prostate cancer: efficacy and safety in clinical practice. Oncol Lett 17:1467-1476. https://doi.org/10.3892/ol.2018.9785

24. De Vincentis G, Frantellizzi V, Follacchio GA et al (2019) No evidence of association between psychological distress and pain relief in patients with bone metastases from castration-resistant prostate cancer treated with 223 Radium. Eur J Cancer Care. https ://doi.org/10.1111/ecc.13112

25. De Vincentis G, Monari F, Baldari S et al (2018) Narrative medicine in metastatic prostate cancer reveals ways to improve patient awareness \& quality of care. Fut Oncol 14:2821-2832. https://doi. org/10.2217/fon-2018-0318

26. Tucci M, Caffo O, Buttigliero C et al (2019) Therapeutic options for first-line metastatic castration-resistant prostate cancer: suggestions for clinical practise in the CHAARTED and LATITUDE era. Cancer Treat Rev 74:35-42. https://doi.org/10.1016/j. ctrv.2019.01.002

27. Nakazawa M, Paller C, Kyprianou N (2017) Mechanisms of Therapeutic Resistance in Prostate Cancer. Curr Oncol Rep 19:13. https://doi.org/10.1007/s11912-017-0568-7

28. Miller K, Gschwend J, Wolff JM (2016) Strategies for first-line treatment of mCRPC. Aktuelle Urol 47:395-401. https://doi. org/10.1055/s-0042-108533

29. Etchebehere EC, Milton DR, Araujo JC et al (2016) Factors affecting (223)Ra therapy: clinical experience after 532 cycles from a single institution. Eur J Nucl Med Mol Imaging 43:8-20. https:// doi.org/10.1007/s00259-015-3185-4 
30. Frantellizzi V, Costa R, Mascia M et al (2019) Primary radical prostatectomy or ablative radiotherapy (RP/EBRT) are protective factors in mCRPC patients treated with 223 Radium-dichloride: an Italian Multicenter Study. Clin Genitourin Cancer. https://doi. org/10.1016/j.clgc.2019.10.009

31. De Vincentis G, Follacchio GA, Frantellizzi V et al (2016) Prostate-specific antigen flare phenomenon during 223 Ra-dichloride treatment for bone metastatic castration-resistant prostate cancer: a case report. Clin Genitourin Cancer 14:e529-e533. https://doi. org/10.1016/j.clgc.2016.04.014

32. Som A, Tu SM, Liu J et al (2012) Response in bone turnover markers during therapy predicts overall survival in patients with metastatic prostate cancer: analysis of three clinical trials. Br J Cancer 107:1547-1553. https://doi.org/10.1038/bjc.2012.436

33. Heinrich D, Bruland O, Guise TA et al (2018) Alkaline phosphatase in metastatic castration-resistant prostate cancer: reassessment of an older biomarker. Fut Oncol 14:2543-2556. https ://doi.org/10.2217/fon-2018-0087

34. Sabbatini P, Larson SM, Kremer A et al (1999) Prognostic significance of extent of disease in bone in patients with androgenindependent prostate cancer. J Clin Oncol 17:948-957

35. Frantellizzi V, Farcomeni A, Follacchio GA et al (2017) A 3-variable prognostic score (3-PS) for overall survival prediction in metastatic castration-resistant prostate cancer treated with 223 Radium-dichloride. Ann Nucl Med 32:142-148. https://doi. org/10.1007/s12149-017-1228-6

36. Graff JN, Beer TM (2014) Pharmacotherapeutic management of metastatic, castration-resistant prostate cancer in the elderly: focus on non-chemotherapy agents. Drugs Aging 31:873-882. https:// doi.org/10.1007/s40266-014-0224-y

37. Tanaka H, Nakashima Y, Ito M et al (2019) Intensity-modulated radiation therapy for elderly patients (aged $\geq 75$ years) with localized prostate cancer: comparison with younger patients (aged $<75$ years). Mol Clin Oncol 10:476-480. https://doi. org/10.3892/mco.2019.1810

38. Tay KJ, Moul JW, Armstrong AJ (2016) Management of Prostate Cancer in the Elderly. Clin Geriatr Med 32:113-132. https://doi. org/10.1016/j.cger.2015.08.001

Publisher's Note Springer Nature remains neutral with regard to jurisdictional claims in published maps and institutional affiliations. 